\title{
THE ROLE OF TECHNOLOGY IN THE INTEGRATION BETWEEN BIOMEDICAL SIGNALS AND BIOIMAGING
}

\author{
SERGIO CERUTTI *, ANNA M. BIANCHI *, GIOVANNA RIZZO **
}

ABSTRACT. - In the last few years the tremendous developments in biomedical technologies made available advanced instrumentation and new data processing techniques able to provide new knowledge on morphology and functional features of internal organs by a multiscale approach. Several techniques are currently available for the examination of the brain under normal and pathological conditions. Magnetic Resonance Imaging (MRI) and x-ray Computed Tomography (CT) supply information about the brain structure. Emission Tomography (PET, SPET) permits the investigation of the functional activity of the brain by measurements of blood flow, metabolism and receptor distribution. The localization of the bio-electric sources of the brain's electric and magnetic fields at the scalp surface can be achieved by EEG and MEG signal analysis.

Correlation of such different information can support the comprehension of the human brain in patho-physiological conditions, since the integration of multimodal information allows us to overcome intrinsic limitations and to enhance the specific potential of each technique. To help forming a correct interpretation of the relation among the different modalities, a unique spatial reference system is defined, into which one must represent images and signals relative to the same subject.

Integration of imaging and signal techniques provides an accurate description of cerebral functions with high spatial and temporal resolution, thus representing a powerful probe for brain investigation both in basic and clinical neuroscience.

\footnotetext{
* Dipartimento di Bioingegneria del Politecnico di Milano.

** IBFM-CNR, Istituto Scientifico Ospedale San Raffaele, Milano.
} 


\section{INTRODUZIONE}

Recenti sviluppi tecnologici hanno ampliato la classe delle tecniche di indagine dell'anatomia e della funzionalità dei diversi organi. Tra di esse si distinguono la Risonanza Magnetica (RM) e la Tomografia Assiale Computerizzata a raggi-X (TAC), che evidenziano le caratteristiche anatomiche e strutturali dei tessuti, la Tomografia ad Emissione di Fotone Singolo (SPET) e ad Emissione di Positroni (PET), che misurano parametri funzionali dei tessuti (quali perfusione, metabolismo, densità recettoriale). A queste possiamo aggiungere la rilevazione segnali relativi ai campi magnetici e/o elettrici, che, nel caso di indagini della funzionalità cerebrale, prendono il nome di MagnetoEncefaloGrafia (MEG) e ElettroEncefaloGrafia (EEG).

Le diverse tecniche si differenziano, oltre che per il tipo di informazioni da esse derivabili, anche per il livello di invasività, per il costo, per la risoluzione spaziale e la risoluzione temporale. Immagini tomografiche, ad esempio, offrono una buona risoluzione spaziale $(<1 \mathrm{~mm}$ nel caso RM), ma una limitata risoluzione temporale (dell'ordine dei secondi/minuti). MEG ed EEG presentano invece una risoluzione temporale dell'ordine del millisecondo a fronte di una più ridotta risoluzione spaziale.

Ciascuna modalità di indagine è dunque caratterizzata da limiti e vantaggi peculiari. Per descrivere, al meglio e nel modo più completo, dal punto di vista spaziale e temporale, diverse strutture anatomiche e la loro funzionalità l'uso integrato di bioimmagini e biosegnali multimodali diventa di fondamentale rilevanza. Solo in questo modo, infatti diventa possibile superare alcuni limiti ed esaltare valori specifici di ciascuna tecnica, per una più accurata interpretazione delle funzioni cerebrali in condizioni fisiologiche o patologiche.

Per ottenere una corretta interpretazione delle informazioni derivate da metodiche differenti, è necessario definire un sistema di riferimento spaziale comune, nel quale rappresentare immagini e segnali relativi allo stesso soggetto. In tale spazio di riferimento, la rappresentazione dell'organo del soggetto costituisce il modello anatomico nel quale riportare le informazioni funzionali multimodali. In tal modo è possibile ottenere una corrispondenza punto a punto delle stesse strutture anatomiche nelle diverse immagini, o localizzare le sorgenti di attività metabolica, funzionale, elettrica o magnetica stimate a partire dai diversi dati. 
Nel seguito, per una più chiara esposizione delle metodiche impiegate, verranno presentati esempi riferiti allo studio dell'anatomia e della funzionalità cerebrale.

\section{INTEGRAZIONE DI SEGNALI E IMMAGINI MULTIMODALI}

Le tecniche tomografiche descrivono il cervello come una sequenza di sezioni contigue che, a seconda della metodica impiegata, possono portare informazioni anatomiche, metaboliche, funzionali. Da esse, in un secondo momento, è possibile ottenere la rappresentazione di un volume tridimensionale riferito ad un sistema di coordinate spaziali specifico del tomografo di acquisizione. L'integrazione dei diversi studi è realizzata con l'uso di tecniche di "registrazione" che permettono il riallineamento dei diversi sistemi di riferimento spaziale mediante stima dei necessari parametri di trasformazione geometrica.

Una delle strategie più comuni alla registrazione di immagini multimodali si basa sull'uso di punti di repere esterni, in genere sorgenti puntiformi o lineari, visibili nelle diverse modalità di acquisizione, fissati sulla pelle o ad un dispositivo di posizionamento del paziente (caschetto) [1, 2, 3]. I markers, visibili nelle diverse modalità, vengono fatti coincidere mediante opportuni algoritmi che determinano la trasformazione geometrica necessaria. Tali trasformazioni vengono realizzate con metodi di rototraslazione (trasformazioni rigide) che portano alla sovrapposizione delle caratteristiche geometriche individuate e, di conseguenza, dei due studi.

Nella Fig. 1a è riportato l'esempio di due studi assiali di RM e PET di uno stesso soggetto con dieci sezioni per la modalità RM e 10 sezioni per la modalità PET. In Fig. $1 b$ ciascuna sezione RM è "sincronizzata e "registrata" nello spazio con le immagini di tipo PET. Ciò permette di visualizzare su un unico supporto informatico sia l'informazione anatomo-morfologico della risonanza sia l'informazione metabolico-funzionale della PET, con indubbi benefici di tipo clinico-diagnostico. Dalle singole immagini tomografiche così "registrate" è possibile la ricostruzione dei relativi volumi, come mostrato in Fig. 1c.

I metodi di registrazione basati su markers esterni presentano il vantaggio di essere indipendenti dalla modalità di acquisizione e dalle alterazioni che alcune patologie possono produrre nelle immagini. L'accuratezza della registrazione è tuttavia vincolata all'accuratezza di riposizionamento dei markers nei diversi studi. 


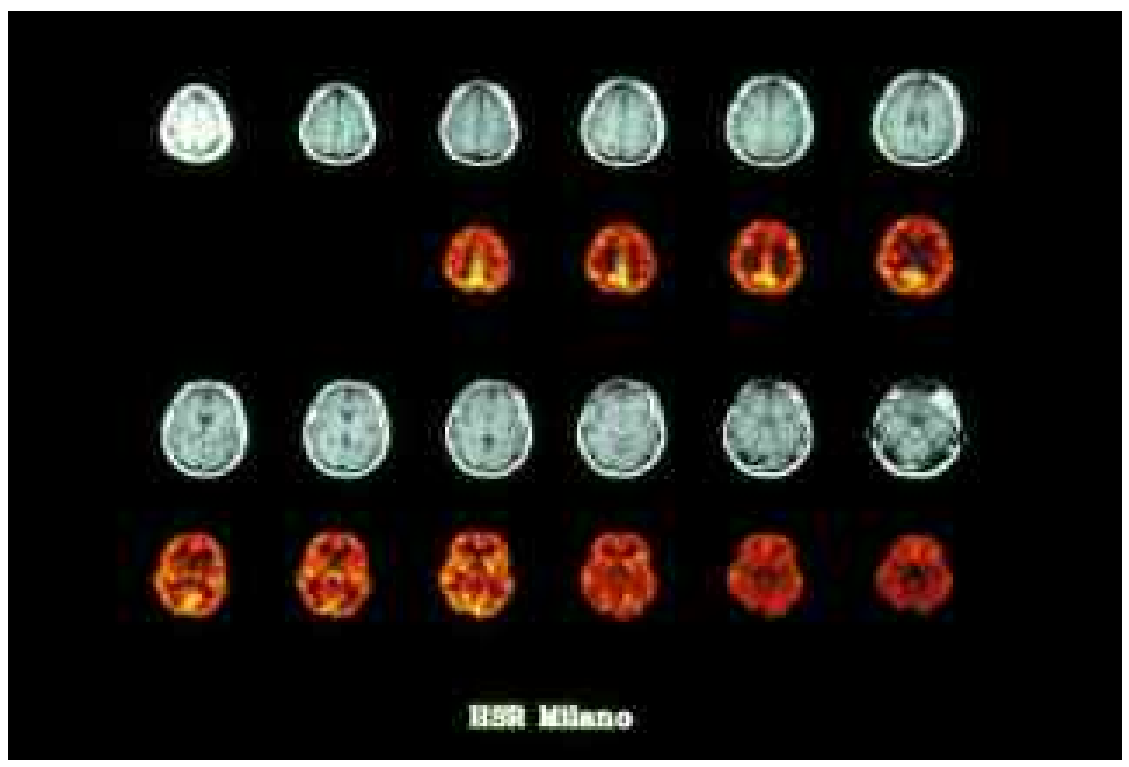

Fig. 1a.

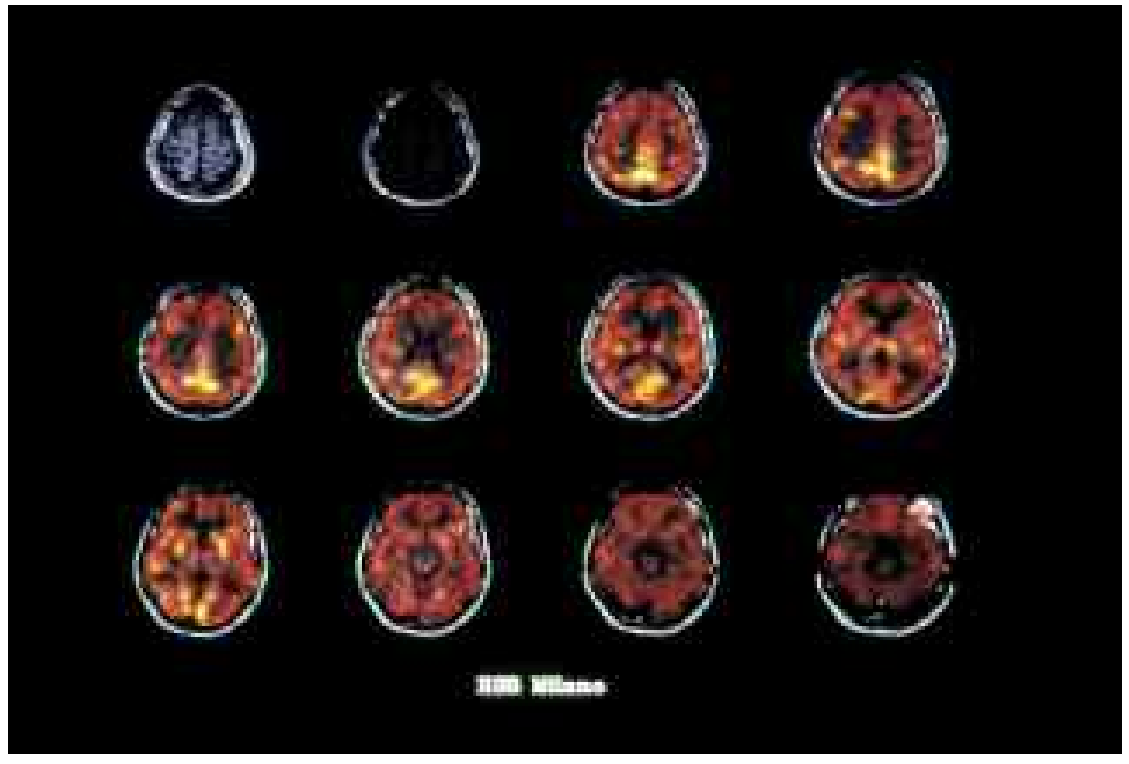

Fig. 1b. 


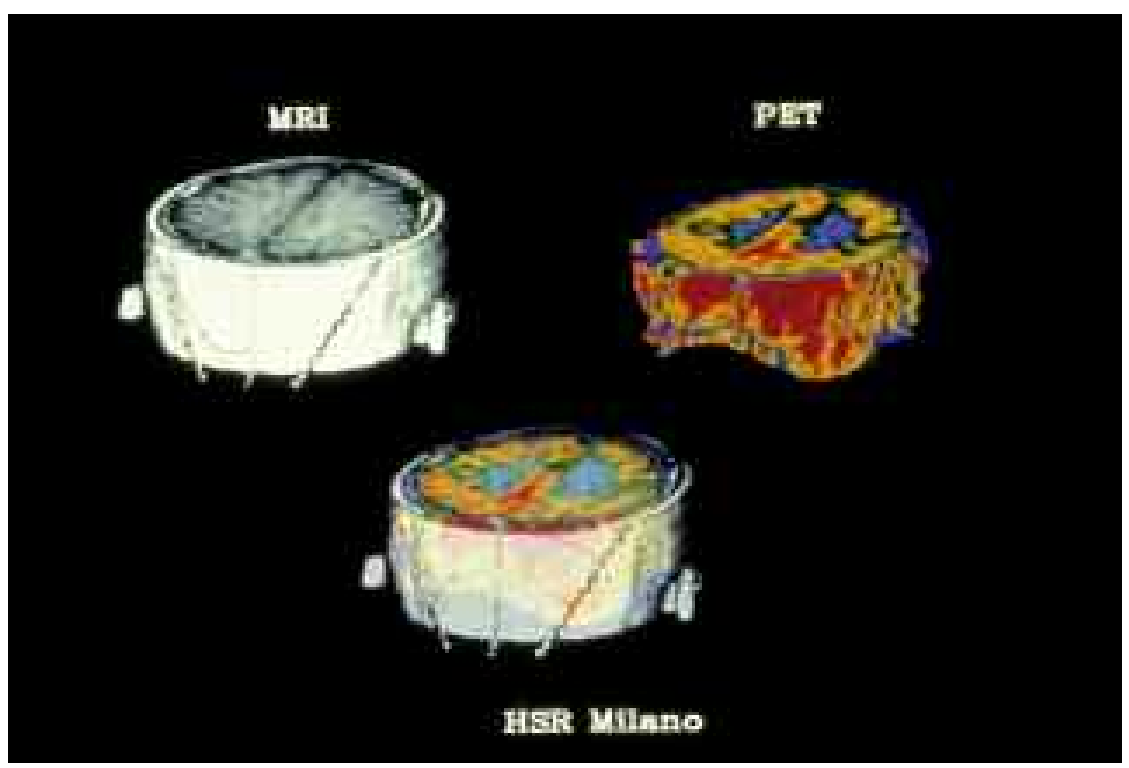

Fig. 1 .

Fig. 1.

a) Immagini cerebrali di risonanza magnetica e PET secondo la direzione assiale e $b$ ) loro registrazione; $c$ ) dalle singole immagini bidimensionali si ricostruiscono $i$ volumi di risonanza magnetica e PET e la loro sovrapposizione.

Un differente approccio alla registrazione delle immagini si basa sulla considerazione che in due studi multimodali dello stesso organo è possibile riconoscere le stesse strutture anatomiche o caratteristiche geometriche omologhe, da cui identificare i diversi sistemi di riferimento spaziale $[4,5,6,7]$. Nel caso del cervello, punti di repere fisiologici possono essere alcune strutture cerebrali o punti anatomici facilmente individuabili sullo scalpo (quali nasion o inion) o nel cervello (quali commissura anteriore e commissura posteriore) o superfici (superficie dello scalpo, superficie del cervello).

I metodi di registrazione sopra citati sono di tipo interattivo e spesso risentono dell'esperienza e dell'oggettività dell'utilizzatore. Tali limitazioni possono essere superate mediante tecniche automatiche, basate sull'analisi pixel a pixel delle immagini $[8,9]$. Tali tecniche di registrazione hanno però una buona efficacia solo se due diversi studi da 
riallineare presentano le stesse caratteristiche morfologiche. L'efficacia del metodo è quindi vincolata alla confrontabilità intrinseca delle informazioni presenti nelle immagini da registrare. Per questo motivo, tale approccio è particolarmente indicato per la registrazione di studi acquisiti con la stessa modalità, per esempio per integrare le informazioni anatomiche rilevate mediante RM e immagini funzionali ottenute con la stessa metodica (fRM). La Fig. 2 mostra, in sette sezioni coronali, la rappresentazione delle aree cerebrali attivate dal movimento volontario del dito, e messe in evidenza tramite fRM, rappresentate sul modello anatomico dello stesso soggetto ottenuto con scansioni RM.

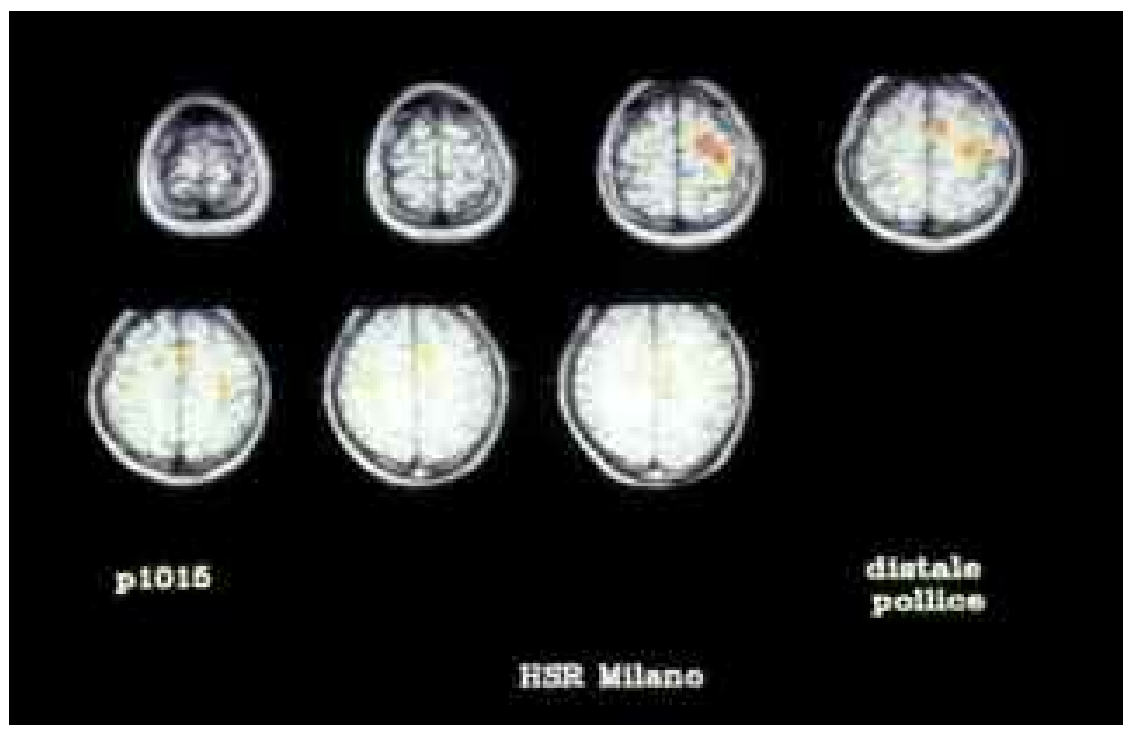

Fig. 2.

Sette sezioni coronali, la rappresentazione delle aree cerebrali attivate dal movimento volontario del dito, e messe in evidenza tramite fRM, rappresentate sul modello anatomico dello stesso soggetto ottenuto con scansioni RM.

Le tecniche di registrazione illustrate finora, basate su trasformazioni geometriche lineari, sono adatte all'integrazione di studi multimodali relativi alla stesso soggetto. In ambito clinico e di ricerca è utile spesso riferire studi relativi a soggetti diversi ad un atlante di riferimento, che rappresenti uno spazio neuroanatomico standard (ad esempio l'atlante di Talairach). Per adattare l'anatomia del soggetto all'atlante di riferi- 
mento è necessario ricorrere a trasformazioni geometriche di tipo non lineare che correggano le variazioni anatomiche interindividuali. Tali trasformazioni sono più costose dal punto di vista computazionale rispetto a quelle lineari, ma permettono di compensare meglio le differenze morfologiche presenti $[10,11,12,13]$.

\section{MAPPAGGIO DELL’ATTIVITÀ ELETTROFISIOLOGICA}

Uno strumento molto diffuso per l'indagine della funzioanalità cerebrale è l'elettroencefaligrafia che consente di rilevare in modo del tutto non invasivo l'attività elettrica del cervello (spontanea o evocata da opportuni stimoli sensoriali) mediante elettrodi posti sullo scalpo. Attualmente le acquisizioni del segnale vengono effettuate utilizzando numerosi canali (20 per la routine clinica, fino a 128 per scopi di ricerca) che vanno a campionare i diversi siti cerebrali e rendono quindi possibile una valutazione globale della funzionalità corticale. La Fig. $3 a$ mostra un esempio di registrazione del segnale EEG come misura della differenza di potenziale elettrico tra due elettrodi posti sullo scalpo. Una tecnica di visualizzazione dell'attività elettrica, ormai molto diffusa, è quella del mappaggio del segnale. Consiste nel fissare un istante temporale di interesse, come viene mostrato nella Fig. 3b, per esempio in corrispondenza di particolari onde sulle risposte evocate, e nel riportare i valori di potenziale corrispondenti su una mappa degli elettrodi come mostrato in Fig. 3c. I valori del potenziale nei punti della mappa non corrispondenti agli elettrodi vengono ottenuti mediante interpolazione. Le tecniche di interpolazione generalmente impiegate possono essere basate su metodiche lineari, che presentano il vantaggio di un basso costo computazionale, o su metodiche di tipo spline, che, a fronte di un più elevato onere computazionale, garantiscono maggiore regolarità dei valori stimati. I valori così ottenuti vengono rappresentati mediante una scala di falsi colori, come mostrato in Fig. 3c.

Una ulteriore fusione di dati può essere realizzata integrando queste mappe con altre informazione ottenibili dalle altre tecniche di imaging, per esempio andando a rappresentare i valori di potenziale elettrico su superfici tridimensionali ottenute dalla reale anatomia del paziente mediante RM o TAC. La Fig. $4 a$ mostra una fusione di dati di risonanza magnetica e di mappe di attività EEG (20 canali rilevati sul cranio nelle classiche posizioni 10-20). 

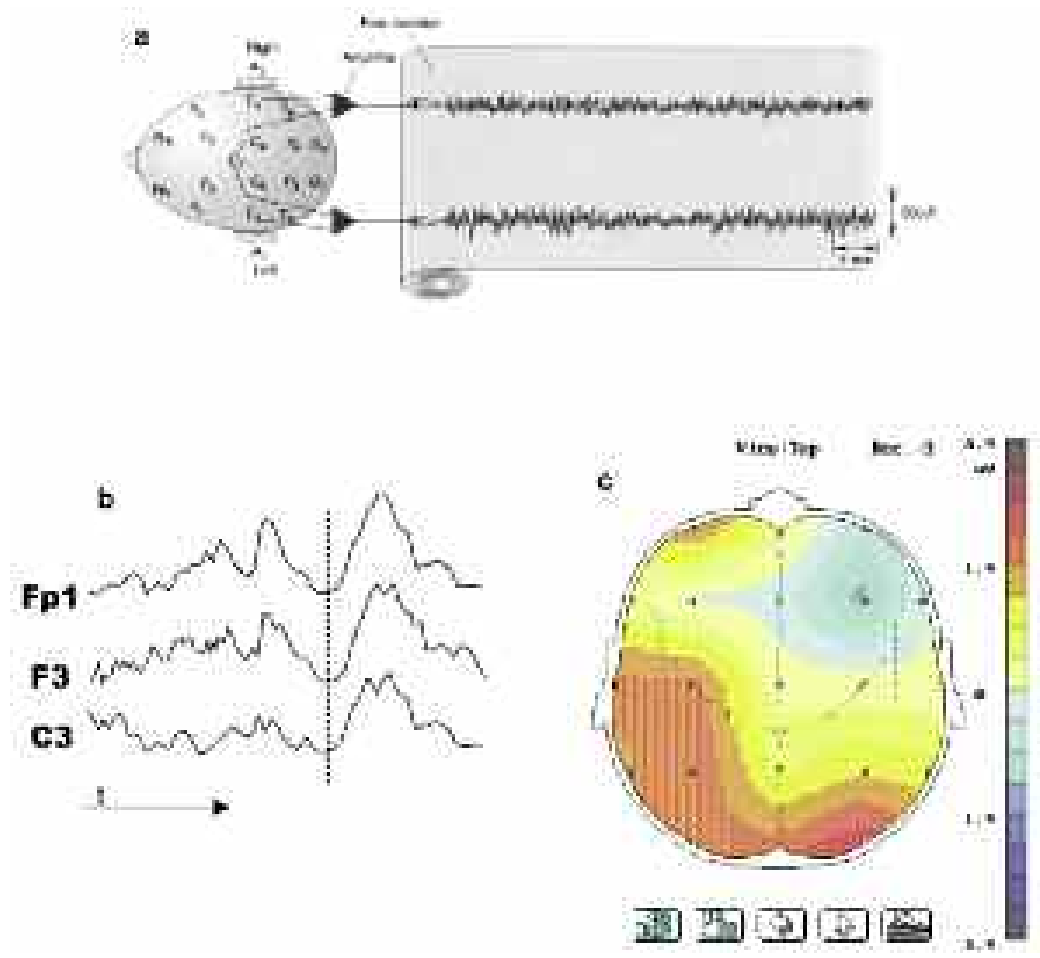

Fig. 3.

a) Il segnale EEG multicanale viene ottenuto come la differenza di potenziale tra diverse coppie di elettrodi posti sullo scalpo. Nella figura sono rappresentati i 20 elettrodi impiegati nel sistema di acquisizione internazionale 10-20. b) Fissato un istante temporale si ottiene un campionamneto spaziale del segnale in corrispondenza della posizione degli elettrodi. Opportune tecniche di interpolazione forniscono una sima del valore del potenziale su tutta la superficie dello scalpo. c) la distribuzione topografica del potenziale viene rappresentata mediante una scala di falsi colori su una superficie bidimensionale.

La mappa è stata ottenuta in corrispondenza dell'onda $\mathrm{P} 40$ di un potenziale evocato somatosensoriale.

Tuttavia l'attività elettrica rilevabile sullo scalpo fornisce una visione attenuata e sfuocata dei fenomeni generati a livello della corteccia cerebrale. Tramite opportuni algoritmi di elaborazione (filtraggio laplaciano e procedure di "deblurring" [14]) è possibile dalla distribuzione del potenziale sulla teca cranica ricostruire il potenziale a livello corticale, come mostrato in Fig. $4 b$ (rimuovendo in modo virtuale lo scalpo e la teca cranica). 


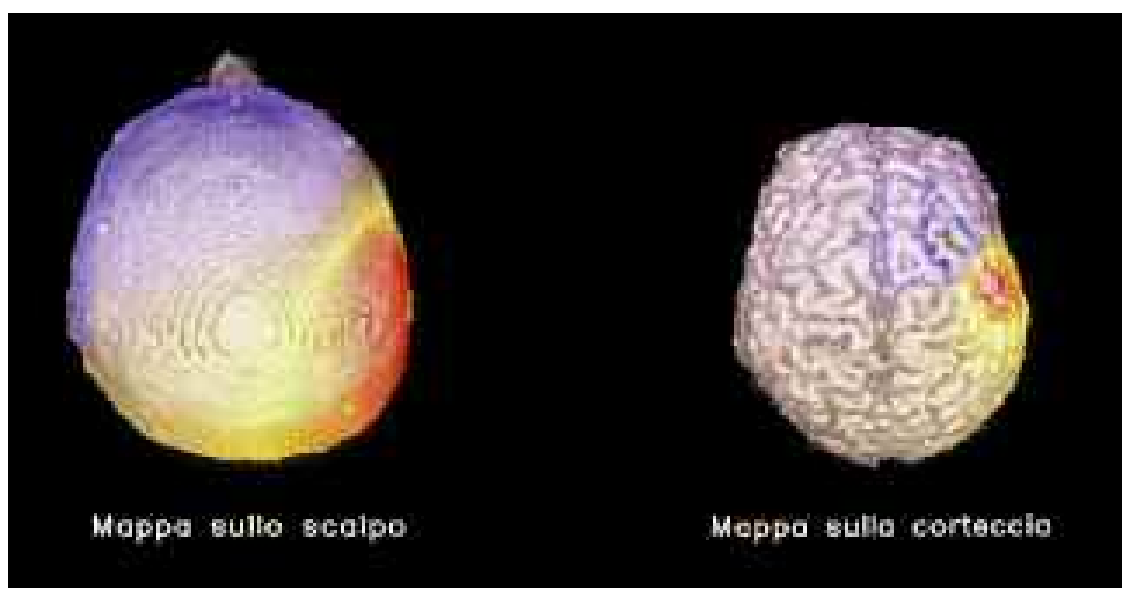

Fig. 4.

a) Mappa di potenziale elettrico, ottenuta in corrispondenza dell'oda P40 di un potenziale evocato somatosensoriale rappresentata sulla superficie realistica dello scalpo; b) la mappa corrispondente a livello corticale rappresentata sulla superficie realistica della corteccia.

Dalla distribuzione dei potenziali sullo scalpo e sulla corteccia, è possibile, sotto opportune ipotesi e vincoli, che tengano in considerazione le caratteristiche anatomiche e fisiologiche del sistema, stimare il vettore momento di dipolo equivalente (cioè la sorgente del potenziale elettrico misurato) che viene localizzato in modo preciso all'interno della struttura cerebrale come punto di applicazione, direzione, modulo e verso. Una precisa allocazione della posizione del vettore all'interno del sistema nervoso centrale può essere ottenuta andando a "registrare" le immagini ottenute dalla risonanza con quelle di atlanti classificati come quelli di Damasio o Talairach [8].

La localizzazione anatomica di sorgenti elettriche, stimate tramite EEG come dipoli di corrente, viene generalmente eseguita presupponendo un opportuno modello geometrico della testa e delle strutture cerebrali. Il modello più comunemente utilizzato è quello sferico o ellissoidale $[15,16,17]$. Il modello sferico può poi essere adattato alla reale anatomia del soggetto per quanto riguarda le dimensioni e le caratteristiche elettriche per una più realistica rappresentazione in corrispondenza delle strutture che ne costituiscono la sorgente, come è mostrato in Fig. 5. La localizzazione dei dipoli EEG nell'anatomia reale del soggetto permette un'associazione diretta tra fenomeni elettrici e aree cerebrali coinvolte. 


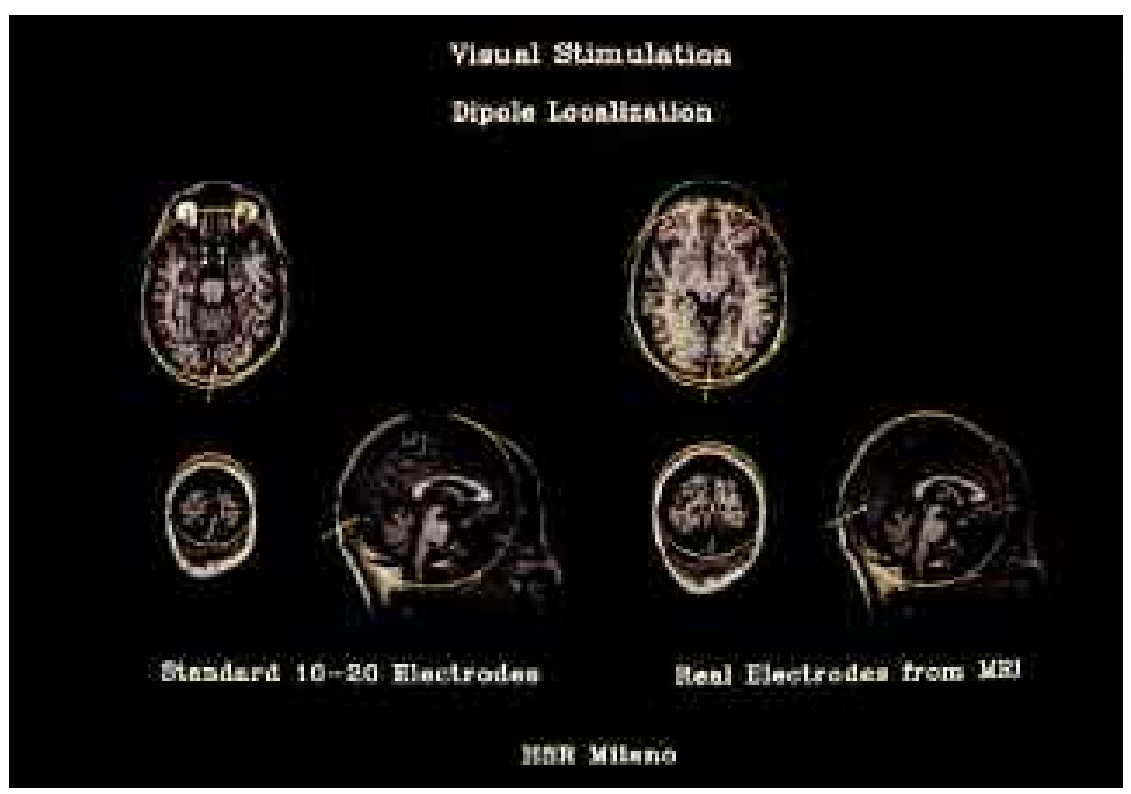

Fig. 5 .

Il dipolo equivalente stimato mediante modello sferico della testa viene rappresentato sull'anatomia realistica della testa.

Per una localizzazione più precisa l'informazione morfologica derivata da studio RM può essere inclusa nel processo di stima dei dipoli EEG, con un conseguente miglioramento nell'accuratezza del posizionamento anatomico.

Nel caso di integrazione EEG/RM, come punti di repere possono essere utilizzati marker anatomici sullo scalpo, identificati dalle immagini RM, e markers esterni EEG, fissati in fase di acquisizione nelle stesse posizioni anatomiche (posizione degli elettrodi).

È perciò necessario, ogni volta, identificare la posizione spaziale di tutti gli elettrodi, per poterla riportare correttamente sul volume RM. Un semplice metodo di registrazione è quello basato sull'uso di markers esterni RM, posizionati negli stessi punti anatomici utilizzati per gli elettrodi EEG. Dal punto di vista pratico tale approccio presenta una certa complessità, dovuta alla necessità di un protocollo di acquisizione rigido (acquisizione EEG seguita, a breve distanza di tempo, da acquisizione RM) e di un numero di markers corrispondente al numero di elettrodi EEG. 
La disponibilità di sistemi di acquisizione EEG a più canali, rispetto ai classici sistemi a 21 elettrodi, può rendere impraticabile, in particolare dal punto di vista clinico, tale approccio di registrazione. Un metodo alternativo si basa sull'uso di sistemi di riconoscimento spaziale (ad es. a penna ottica), in grado di campionare rapidamente la superficie dello scalpo ed in particolare la posizione degli elettrodi EEG, per la generazione di un modello scalpo-EEG. La registrazione tra EEG ed RM è quindi ottenuta con l'impiego di metodi di minimizzazione che portano alla sovrapposizione del modello scalpo-EEG allo scalpo come visualizzato nelle immagini RM.

Fusioni di dati ugualmente importanti si possono ottenere da potenziali elettrici evocati e dalla PET o dalla fMR, ad esempio andando a valutare l'effetto congiunto di una stimolazione di tipo cognitivo sia sulla mappa PET o fMR che sulla distribuzione del potenziale a livello corticale. Tale tipo di indagine porterà ad importanti considerazioni sul funzionamento del cervello e potrà rendere più "oggettivo" l'approccio del clinico per la valutazione di deficit cognitivo e/o comportamentale.

La Fig. 6 mostra uno studio in cui sono state integrate le informazioni funzionali derivare da una registrazione EEG e rappresentate come sorgente equivalente, con le informazioni metaboliche derivanti da uno studio PET. Queste sono state poi rappresentate nella reale anatomia del soggetto ottenuta dalla ricostruzione 3D del volume della testa a partire da immagini tomografiche RM.

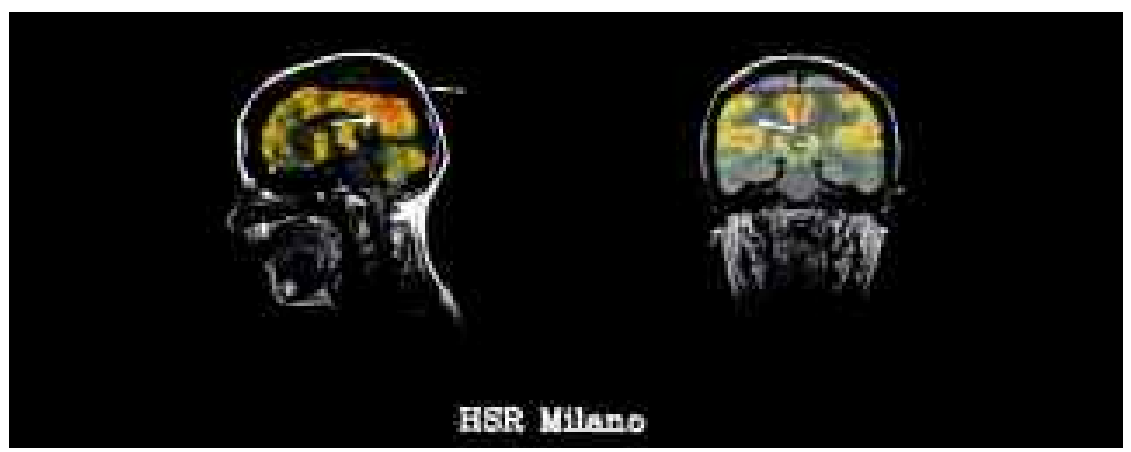

Fig. 6.

Studio di integrazione tra le informazioni funzionali derivare da un esame EEG $e$ rappresentate come sorgente equivalente, con le immagini metaboliche derivanti da uno studio PET. Queste sono state poi rappresentate nella reale anatomia del soggetto ottenuta dalla ricostruzione $3 D$ del volume della testa a partire da immagini tomografiche $R M$. 


\section{CONCLUSIONE}

Uno degli obiettivi principali dell'Ingegneria Biomedica è quello di rendere disponibili metodi di elaborazione dei dati e delle immagini atti ad integrare le diverse informazioni in essi contenuti, oltre a elaborare modelli di interpretazione delle complesse realtà biologiche per affrontare in modo più oggettivo e organico il problema del miglioramento delle conoscenze fisiologiche e delle applicazioni nella clinica diagnostica. Sono stati introdotti alcuni esempi significativi di come metodiche più o meno avanzate nel settore della elaborazione delle immagini e dei segnali possono contribuire a migliorare le conoscenze del sistema nervoso centrale. Tecniche analoghe possono essere applicate all'indagine di altri distretti anatomi-funzionali, quali il cardiovascolare, il cardiorespiratorio, quello endocrino-metabolico, etc. Molti degli esami che vengono condotti attualmente vengono realizzati in diversi reparti dell'ospedale, a volte con scarsa comunicazione ed interazione. Non c'è dubbio che le tecnologie biomediche possono integrare in un unico supporto informatico tutte le informazioni che riguardano lo stesso paziente. Questa operazione deve essere vista non solo come una razionalizzazione che l'automazione in genere deve comportare, ma anche come opportunità per realizzare una vera e propria "fusione" dei dati e delle immagini con inferenza delle informazioni ottenute per tramite degli algoritmi multimodali sopra descritti. In questo modo è possibile recuperare anche una "centralità" del paziente che viene visto come un "unicum" e non come una semplice collezione di segnali e immagini, e questo comporta un indubbio beneficio dal punto di vista medico e sociale.

\section{BIBLIOGRAFIA}

[1] Rizzo, G., Gilardi, M.C., Prinster, A., Lucignani, G., Bettinardi, V., Triulzi, F., Cardaioli, A., Cerutti, S. and Fazio, F. ( 1994) A bioimaging integration system implemented for neurological applications, J. Nuc. Biol. Med. 38(4), 579-85.

[2] Maisey, M.N., Hawkes, D.J. and Lukawiecki-Vydelingum, A.M. (1992) Synergistic imaging, Eur. J. Nucl. Med. 19, 1002-1005.

[3] Hawkes, D.J., Hill, D.L.G., Lehmann, E.D., Robinson, G.P., Maisey, M.N. and Colchester, A.C.F. (1990) Preliminary work on the interpreta- 
tion of SPECT images with the aid of registered MR images and an MR derived 3D neuro-anatomical atlas, in $\mathrm{H}$. Hoene, S. M. Pizer and H. Fuchs (eds.), 3D Imaging in Medicine, Nato ASI Series F 60 K, SpringerVerlag, Berlin, pp. 241-252.

[4] Evans, A.C., Marrett, S., Collins, L. and Peters, T.M. (1989) Anatomicalfunctional correlative analysis of the human brain using three dimensional imaging system, in R.H.Schneider, S.J. Dwyer III, R.Gilbert Jost (eds.), Proceedings of SPIE Medical Imaging III: Image processing, SPIE Press, Bellingham W.A. 1092, pp. 264-274.

[5] Pelizzari, C.A. ( 1998) Image processing in stereotactic planning: volume visualization and image registration, Med Dosim 23(3), p. 137-45.

[6] Pelizzari, C.A., Chen, G.T.Y., Spelbring, D.R., Weichselbraum, R.R. and Chen C.T. (1989) Accurate Three-Dimensional Registration of CT, PET and/or MR Images of the Brain, J. Comput. Assist. Tomogr. 13, 20-26.

[7] Woods, R.P., Cherry, S.R. and Mazziotta, J.C. (1992) Rapid automated algorithm for aligning and reslicing PET images, J. Comput. Assist. Tomogr. $16,620-633$.

[8] Hoh, H.K., Dahlbom, M., Harris, G., Choi, Y., Hawkins, R.A., Phelps, M.E. and Maddahi, J. (1993) Automated iterative three-dimensional registration of positron emission tomography images, J. Nucl. Med. 34, 2009-2018.

[9] Rizzo, G. Scifo, P., Gilardi, M. C., Bettinardi, V., Grassi, F., Cerutti, S. and Fazio, F. ( 1997) Matching a computerized brain atlas to multimodal medical images, Neuroimage 6(1), 59-69.

[10] Bajcsy, R. and Kovaci S. (1989) Multiresolution elastic matching. Computer Vision, Graphics and Processing 46, 1-21.

[11] Bookstein, F.L. (1989) Principal Warp: Thin plate spline and the decomposition of deformation, IEEE Trans. PAMI 567-585.

[12] Evans, A.C., Dai, W., Collins, L., Neelin, P. and Marrett, S. (1991) Warping of a computerized 3-D atlas to match brain image volumes for quantitative neuroanatomical and functional analysis. In Loew $\mathrm{MH}$, ed. Medical imaging V: image processing. Washington, DC: SPIE 1652, 226-34.

[13] Gevins A., Le J., Leong H., McEvoy L.K. and Smith M.E. (1999) Deblurring, J Clin Neurophysiol. 16(3), 204-13. 
[14] Romani, G.L. and Pizzella, V. (1990) Localization of brain activity with magnetoencephalography, in S. Sato (ed.), Advances in Neurology: Magnetoencephalography Vol. 54, Raven Press, New York, pp. 67-78.

[15] Tatcher, R.W. (1995) Tomographic electroencephalography-magnetoencephalography: dynamics of human neural network switching, J. Neuroimag. 5, 35-45.

[16] Gevins, A., Cutillo, B., DuRousseau, D., Le, J., Leong, H., Martin, N., Smith, M.E., Bressler, S., Brickett, P., McLaughlin, J., Barbero, N. and Laxer, K. [1994] Imaging the spatiotemporal dynamics of cognition with high-resolution evoked potential methods, Human Brain Mapping 1, 101-116. 